\title{
Recurrent bronchiectasis in adults due to an abnormal bronchus
}
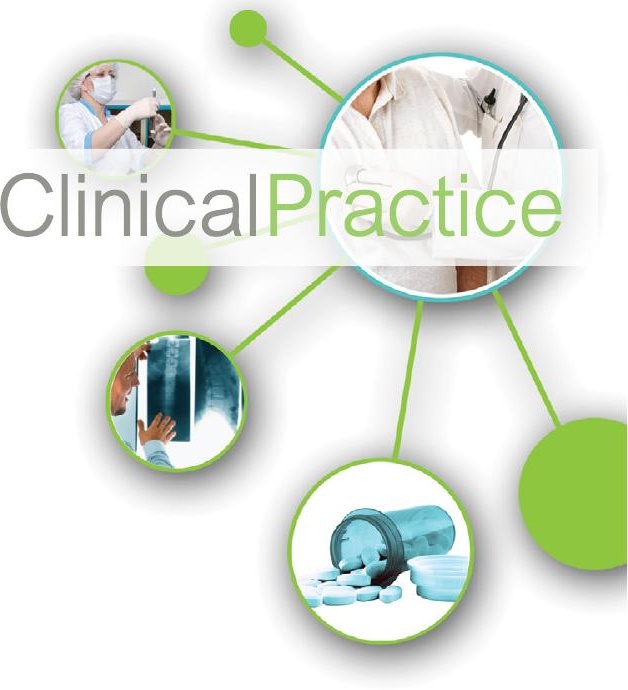

\begin{abstract}
The tracheal bronchus is a rare tracheobronchial anomaly, the average incidence of $0.1 \%-2 \%$, most commonly discovered in childhood due to its association with congenital syndromes. Occasionally during late adulthood, it manifests in the form of chronic recurrent infections. We are describing a case of atypical manifestation of tracheal bronchus. The patient presented late during her life with symptoms of recurrent respiratory symptoms. Her condition was missed on radiological imaging and was identified on bronchoscopy. After the root cause was evaluated, she was referred for lobectomy/ segmentectomy for definitive treatment.
\end{abstract}

Keywords: tracheal bronchus, bronchiectasis, respiratory, lung

\section{Introduction}

The true tracheal bronchus is an aberrant bronchus arising above $2-6 \mathrm{~cm}$ from carina, from the lateral wall of trachea and directed towards the upper lung lobe [1]. Frequently located on the right side, it can be further described as supernumerary if there is an anatomic right bronchus with normal segmental apical, anterior and posterior bronchi. It is considered displaced if it's accompanied by an absence of anatomic segmental bronchi. Tracheal bronchus can supply the entirety or part of the upper lung parenchyma. The incidence described in the literature ranges from $0.1 \%$ to $1 \%$ reported in different studies. Prevalence of right tracheal bronchus has been reported as $0.1 \%-2 \%$ and left tracheal bronchus a prevalence of $0.3 \%-1 \%$ on bronchographic and bronchoscopic studies [2]. However, the incidence of $0.31 \%$ was reported via Multi-Detector Computed Tomography (MDCT) analysis in a study of $9 \mathrm{k}$ cases. With the advent of advanced imaging techniques, its detection has become easier [3]. We described a rare presentation of tracheal bronchus in late adulthood in the form of recurrent respiratory infections.

\section{Case Description}

We present a case of a 59-year-old, nonsmoking, Caucasian female of Eastern European descent. She had a past medical history significant for chronic bronchiectasis and peptic ulcer disease. She was presented to our clinic with complaints of pleuritic chest pain associated with productive cough. The patient had similar symptoms in the past and was diagnosed with bronchiectasis in 2014 via a CT scan of the chest. She had undergone a bronchoscopy during that time, which was non-diagnostic. Since then, the patient's home medications included a regimen of albuterol, long-acting beta-agonist, and inhaled corticosteroids, which controlled her respiratory symptoms.

Upon examination, her vital signs were found to be stable with an $\mathrm{O}_{2}$ saturation of $97 \%$ on room air. Lung auscultation revealed diffuse rhonchi bilaterally. The rest of the physical examination was unremarkable.

Given the patient's history of chronic bronchiectasis previously controlled but now unresponsive to LABA/ICS, a CT scan of the chest with contrast was done. Compared to her previous CT scan in 2014, new peribronchial opacities were noted in the right upper lobe. Flexible bronchoscopy with Bronchoalveolar Lavage (BAL) was performed. Findings revealed normal vocal cords, severe erythema and inflammation of the bronchial mucosa with purulent mucus secretions and an accessory

\section{Asma Jamil ${ }^{1 *}$, Rajesh Gulati ${ }^{1}$ and Ayesha Jamil ${ }^{2}$}

${ }^{1}$ Department of Internal Medicine, Riverside Community Hospital, USA

${ }^{2}$ Department of Internal Medicine, St. Mary Hospital, USA

*Author for correspondence: Jamilasma40@gmail.com 
bronchus arising within $2 \mathrm{~cm}$ proximal to the carina on right lateral wall, at the 9 and 10 o'clock position (as shown by blue arrows in FIGURE 1). The opening of the bronchus was approximately $8-10 \mathrm{~mm}$, and the scope was successfully introduced into the bronchus for $1 \mathrm{~cm}$. Saline lavages into the bronchus returned with saline and air bubbles on multiple attempts, confirming the presence of communication between the tracheal bronchus and lung parenchyma. BAL was negative for fungal culture. AFB smear and 6-week culture, chlamydial PCR, viral culture, mycoplasma antibody, legionella direct fluorescent antibody were negative. However, the BAL gram stains and cultures grew pan-sensitive Pseudomonas aeruginosa. The patient was started on levofloxacin.

CT chest with $3 \mathrm{D}$ reconstruction was ordered but due to lack of availability, High-Resolution
Computed Tomography (HRCT) was performed, which showed persistent bilateral bronchiectasis. Subsequent imaging with HRCT and bronchoscopies was stable after the initiation of long-acting beta-agonists, long-acting muscarinic antagonists, inhaled corticosteroids, and antibiotics. However, the symptoms of productive cough with sputum persisted, despite therapy. The patient was referred to and is still following up with the infectious disease specialist for cyclic antibiotic treatment. The patient was referred to cardiothoracic surgery for upper lobe segmentectomy or lobectomy as definitive treatment.

\section{Discussion}

Tracheal bronchus, also called "bronchus suis" is a normal finding in pig and other ruminant animals such as cattle, sheep, and giraffes [3]. True tracheal bronchus, in most cases described in the literature, was found as an incidental

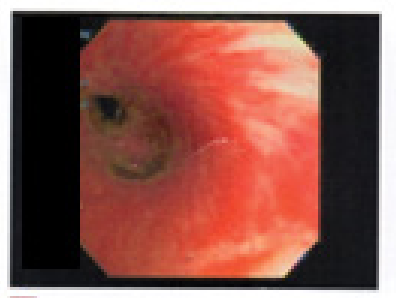

I.

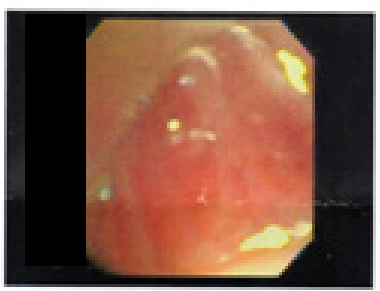

(3)

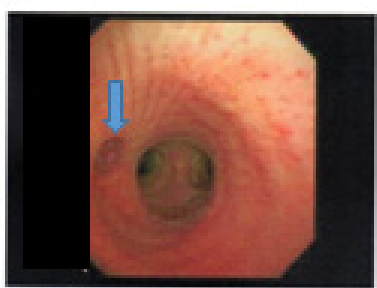

9

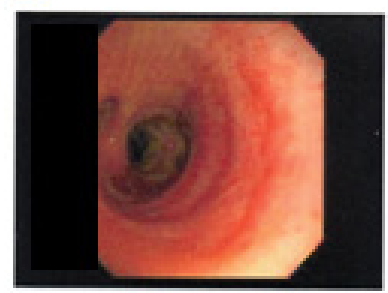

De

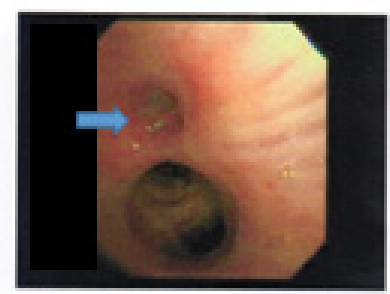

[2]

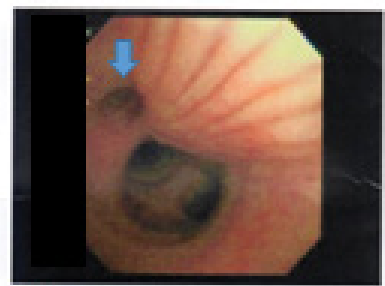

6

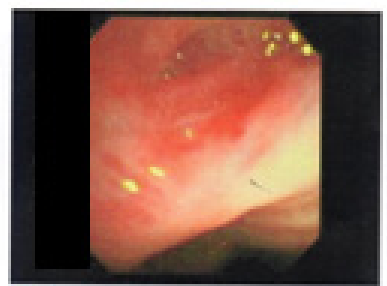

901

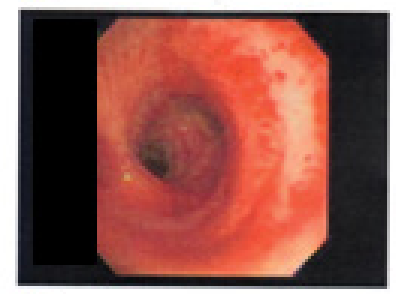

19

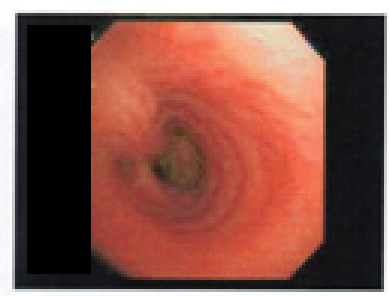

3

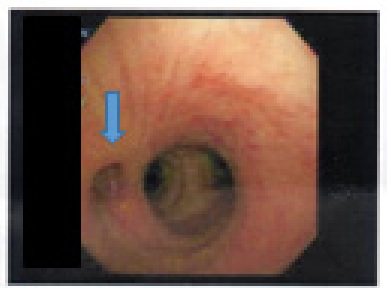

[1]

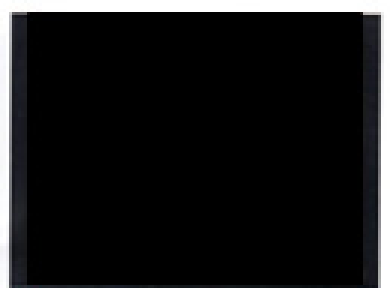

D9.

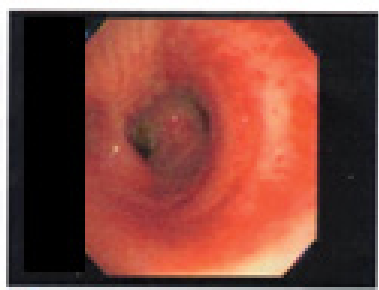

IS

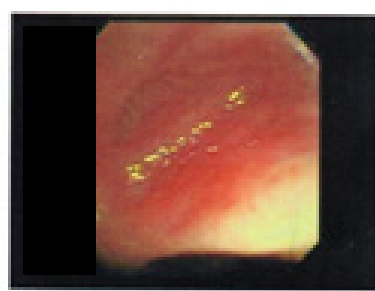

흐
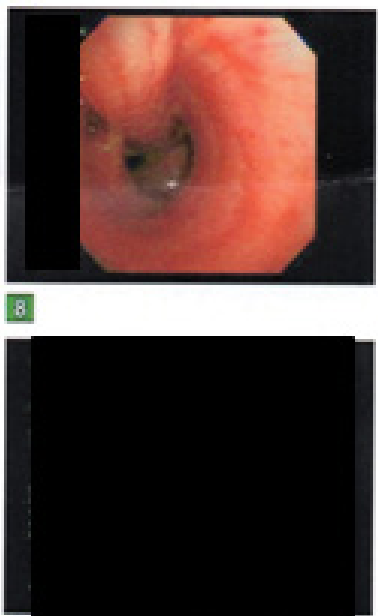

12

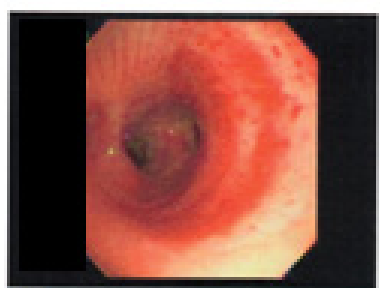

99

FIGURE 1. Arrows points towards origin of tracheal bronchus at 9, 10 O' clock positions above the carina. 
finding during or after intubation [4]. Tracheal bronchus has been frequently found in children where it manifests early in the form of recurrent respiratory infections. In children, it is found as a part of multiple congenital abnormalities e.g. tracheoesophageal fistula, tracheal stenosis, Tetralogy of Fallot, VATER syndrome, and Down's syndrome [5]. Other incidental findings include squamous cell cancer, large cell cancer, tuberculosis, leiomyoma, bronchial carcinoid, and actinomycosis [6-9].

Tracheal bronchus formation is explained via various embryological theories i.e. reduction theory suggests it results from the reduction of a previously developed bronchus. Migration theory explains it to be extension or migration of a part of the developed hyparterial branching pattern to a different location either on trachea or bronchus. Selection theory postulates that local morphogenesis disturbances cause bronchial abnormalities, claiming that bronchus can arise if the bronchial mesenchyme comes in contact with the tracheal epithelium. The underlying cause is usually a congenital malformation. Many theories have been proposed including selection theory, reduction theory, and migration theory [10].

One classification categorizes the tracheal bronchus in relation to the carina anatomically: Type I is when the tracheal bronchus is more than $2 \mathrm{~cm}$ from carina and the distal trachea is narrowed; Type II is when the tracheal bronchus is more than $2 \mathrm{~cm}$ from the carina but the distal trachea is not narrowed; Type III is when the tracheal bronchus is less than $2 \mathrm{~cm}$ above the level of carina [10].

An additional classification system suggested describes tracheal bronchi in relation to the upper lobe bronchus and pulmonary arteries. A normal right upper lobe bronchus is called eparterial as it arises cranial to the right pulmonary artery. A normal left upper lobe bronchus is called hyparterial as it arises caudal to the normal left pulmonary artery. An aberrant bronchus arising proximal to the upper lobe bronchus is called preeparterial on the right side and eparterial or prehyparterial on the left side. A bronchus arising distal to the upper lobe bronchus is called post eparterial on the right side and post hyparterial on the left side $[1,10]$.

Tracheal bronchus may be displaced or supernumerary. If the anatomic upper-lobe bronchus is missing a single branch, the tracheal bronchus is defined as displaced, if the right upper-lobe bronchus has a normal trifurcation into apical, posterior, and anterior segmental bronchi, the tracheal bronchus is defined as supernumerary. The supernumerary bronchi may end blindly; in that case, they are also called tracheal diverticula. If they end in aerated or bronchiectatic lung tissue, they are termed apical accessory lungs or tracheal lobes [6].

It can be symptomatic if there is any aberrancy in venous drainage or ventilation. In that case, it can present itself as recurrent infections, wheezing, and atelectasis. Such a presentation in late adulthood has been described in few other cases such as Panzironi et al. described a case where 75-year-old women presented with persistent productive cough after failed antibiotics treatment. Her CT chest performed to see appropriate resolution showed incidental tracheal bronchus [11].

Our patient had recurrent infections leading to frequent hospitalization. Antibiotic treatment courses were given without any underlying known etiology. Successive CT scans showed the re-demonstration of worsening right middle and upper lobe infiltrates. A careful review of CT scan chest shows tracheal bronchus at the T2T3 level at the level of pulmonary vessels next to the main trachea (FIGURE 2). Bronchoscopy revealed the tracheal bronchus and confirmed its communication with the lung parenchyma.

It's not clear why the patient remained asymptomatic during a major part of her life. Her current symptoms might be the result of abnormally narrowed tracheal bronchus, impairing the ventilation process. A study comparing the airflow distribution between normal lung and tracheal bronchus subjects revealed decreased aeration in comparison to the rest of the lung. High airflow velocity, wall pressure, and wall shear stress are observed locally at the tracheal bronchus. In comparison to healthy controls, the ratio of airflow into the tracheal bronchus accounts for 6.6\%-15.6\% of the inhaled air flow, decreasing the ratio to the right upper lobe airflow from 15.7\%-21.4\% to $4.9 \%-13.6 \%$ [12]. Further investigation is needed to reveal underlying contributing factors.

MDCT with the addition of 3D reconstruction has been the gold standard in diagnosing tracheal bronchus. The $2 \mathrm{D}$ and 3D features helped significantly in identifying and differentiating intra and extra-luminal pathologies in the tracheobronchial tree [13].

Currently, the mainstay of the treatment of 


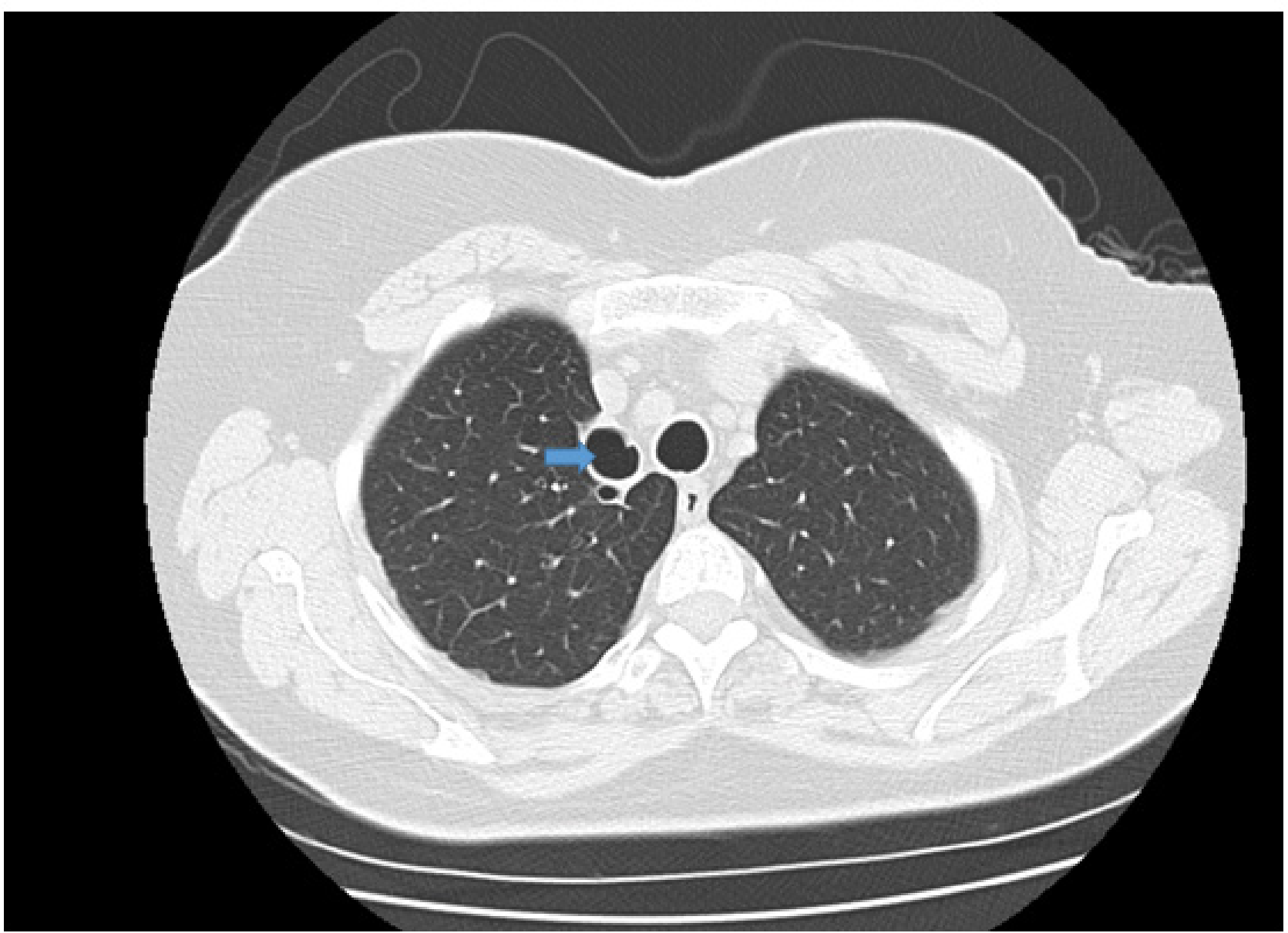

FIGURE 2. Abnormal tracheal bronchus supplying right upper lobe next to the main trachea at level T2-T3.

tracheal bronchus is symptomatic management. Asymptomatic cases require only observation whereas symptomatic tracheal bronchus is treated based on the severity of symptoms. Given the wide range of symptoms that tracheal bronchus can present, medical management includes bronchodilators, inhaled corticosteroids, muscarinic antagonists, and antibiotics. However, if the symptoms are refractory to medication, then surgical treatment is the next step in management. Segmentectomy and lobectomy have been performed for the treatment of recurrent pneumonia resulting from the tracheal bronchus and have been found to be curative [4].

\section{Conclusion}

Adult patients who present with recurrent respiratory infections must be evaluated for rare congenital anomalies like tracheal bronchus. MDCT imaging modalities play a vital role in the identification of tracheobronchial tree anomalies.

\section{Source of Funding \\ None}

\section{Equal Author Contribution State- ment}

All authors have equal contribution to the preparation of this article. 


\section{REFERENCES}

Ghaye B, Szapiro D, Fanchamps JM, et al. Congenital bronchial abnormalities revisited. Radiographics. 21, 105-119 (2001).

Han J, Xiang H, Ridley WE, et al. Pig bronchus. J Med Imaging Radiat Oncol. 62, 34 (2018).

Suzuki M, Matsui O, Kawashima H, et al. Radioanatomical study of a true tracheal bronchus using multidetector computed tomography. Jpn J Radiol. 28, 188-192 (2010).

Conacher ID. Implications of a tracheal bronchus for adult anaesthetic practice. BrJ Anaesth. 85, 317-320 (2000).

Kabra NS, Bowen JR, Allen H. Porcine bronchus diagnosed in neonatal period.
Indian J Pediatr. 68, 681-684 (2001).

Berrocal T, Madrid C, Novo S, et al. Congenital Anomalies of the tracheobronchial tree, lung, and mediastinum: Embryology, radiology, and pathology. Radiographics. 24, 17 (2004).

Nicolaou N, Du Plessis A. Squamous carcinoma arising from a true tracheal bronchus: Management and case report. Int J Surg Case Rep. 6, 256-258 (2015).

Tuon FF, Siqueira AM, Litvoc MN, et al. Tuberculosis and tracheal bronchus. Int J Infect Dis. 11, 467-468 (2007).

Tamura M, Murata T, Kurumaya H, et al. Leiomyoma of an accessory tracheal bronchus. Ann Thorac Surg. 78, 21632165 (2004).

Chassagnon G, Morel B, Carpentier
E, et al. Tracheobronchial branching abnormalities: lobe-based classification scheme. Radiographics. 36, 358-373 (2016).

Panzironi G, Noce V, Ammendola RM, et al. Tracheal bronchus: High resolution computed tomography diagnosis in a symptomatic patient. Eur J Radiol Open. 2, 1-2 (2015).

Qi S, Zhang B, Yue Y, et al. Airflow in tracheobronchial tree of subjects with tracheal bronchus simulated using CT image-based models and CFD method. $J$ Med Syst. 42, 65 (2018).

Laroia AT, Thompson BH, Laroia ST, et al. Modern imaging of the tracheobronchial tree. World J Radiol. 2, 237-248 (2010). 